\title{
Evaluation of Cutting Time and Waste Generation in Different Band Sawmills in Sri Lanka with Special Reference to Teak Timber
}

\author{
Abeykoon A.M.C.*, Amarasekara H.S. \\ Department of Forestry \& Environmental Science, University of Sri Jayewardenepura, \\ Sri Lanka \\ *chanduwijekoon@gmail.com
}

\begin{abstract}
In recent years, due to rising raw material and labor costs, increasing attention is being devoted to improve wood sawing practices. While a high cutting rate is still among the most desired characteristics, other factors such as improved cutting accuracy and surface quality, reduced kerf losses, noise, downtime, and maintenance of the machine are becoming increasingly important. However, band sawmilling have advantages over circular saws which include higher cutting speeds lower kerf waste, and typically lower noise levels. Considering above advantages several Sri Lankan companies have now established band saw milling factories. Thus the key objectives of this study were to evaluate cutting time of band sawmills and to investigate waste generation. Three different band saws (TRAK-MET TTP 600 premium; TM, Wood-Mizer LT20; WM, and Veheran; V) which are commonly used in Sri Lanka were used to this study. Evaluation of cutting time and waste generation in different band sawmills were done with special reference to Teak timber. Results show that the fastest cutting time in WM (mean cutting time of $27 \mathrm{~s}$ ), medium cutting time in TM (mean cutting time of 60s) and the slowest cutting time in Veheran (mean cutting time of 180s). The factors such as tooth profile, setting value, sharpening frequency, status of lubrication, tensioning pressure of the blade and capacity and the condition of the machine may have affected to the fastest cutting time. According to the results of evaluation of saw dust Veheran showed the highest wastage owing to high kerf and factors such as poor lubrication, lower tensioning pressure. Moreover, cutting time negatively correlated with the lubrication and saw tension in band sawmills. Therefore, it can be concluded that proper saw mill management is necessary for reduction of timber wastage and increase the efficiency of the industry.
\end{abstract}

Keywords: Band sawmilling, Waste generation, Teak, Lubrication, Tensioning pressure 